\title{
CO cooling rates for clumpy and turbulent molecular clouds
}

\author{
M. Hegmann ${ }^{1}$, W. H. Kegel ${ }^{2}$, and E. Sedlmayr ${ }^{1}$ \\ 1 Zentrum für Astronomie und Astrophysik (ZAA), Technische Universität Berlin, Hardenbergstr. 36, 10623 Berlin, Germany \\ e-mail: [hegmann; sedlmayr] @astro.physik.tu-berlin.de \\ 2 Institut für Theoretische Physik, Johann Wolfgang Goethe-Universität, Postfach 11 19 32, 60054 Frankfurt am Main, Germany \\ e-mail: kegel@astro.physik.tu-berlin.de
}

Received 13 September 2006 / Accepted 16 April 2007

\section{ABSTRACT}

\begin{abstract}
Aims. Based on a stochastic radiative transfer model (SRTM), which accounts for density and velocity fluctuations with a finite correlation length, we have calculated CO cooling rates for a molecular gas at temperatures $T_{\text {gas }}=(10-100) \mathrm{K}$ and densities $n_{\mathrm{H}_{2}}=$ $\left(10^{2}-10^{6}\right) \mathrm{cm}^{-3}$. In particular, we are interested in how the cooling rates are modified by inhomogeneities of the density distribution and a finite correlation length of the turbulent velocity field.

Methods. Assuming spherical symmetry, we solved the generalized radiative transfer equation simultaneously with the rate equations (full NLTE problem). Depending on the temperature assumed, we took up to 18 rotational levels of the CO molecule into account. Results. Our results show that the finite correlation length of the turbulent velocity field has a great influence on the CO cooling rates. In general, the volume averaged cooling rates are noticeably decreasing with an increasing correlation length of the velocity field except for very high CO cloumn densities. The stochastic density fluctuations, on the other side, tend to increase the CO cooling efficiency. For an inhomogeneous stochastic density distribution, cooling by the high rotational lines of $\mathrm{CO}$ is substantially enhanced. Most of the radiation is emitted from cloud regions with higher density than the average. In addition, an inhomogeneous density field reduces the effect of photon trapping, which leads to a further increase of the cooling rate. A comparison of the SRTM results with earlier work of Neufeld et al. (1995, ApJS, 100, 132) and Juvela et al. (2001, ApJ, 563, 853) is given. It turns out that their predictions and findings can be reproduced rather well by choosing the parameters describing the stochastic density and velocity field appropriately.
\end{abstract}

Key words. radiative transfer - ISM: clouds - ISM: molecules

\section{Introduction}

The temperatures of molecular clouds are determined by the thermal balance between cooling by line emission of molecules and atoms and various heating mechanisms like cosmic ray heating or ionization and photoelectric heating due to an incident FUV radiation field. For a wide range of densities and gas temperatures, rotational line emission of $\mathrm{CO}$ and its isotopomere ${ }^{13} \mathrm{CO}$ is the dominant cooling mechanism. Cooling by less abundant molecules like $\mathrm{O}_{2}$ and $\mathrm{H}_{2} \mathrm{O}$ or cooling due to collisions between gaseous particles and the colder dust grains become effective only at densities much higher than $n_{\mathrm{H}_{2}}=10^{4} \mathrm{~cm}^{-3}$. $\mathrm{CO}$ cooling rates have therefore been studied by a number of authors. Goldreich \& Kwan (1974) calculated CO cooling rates based on the Sobolov or large velocity gradient (LVG) approximation for a homogeneous sphere. Goldsmith \& Langer (1978), Neufeld et al. (1995) and Visuso \& Chernoff (1988) continued the work by using updated molecular data and more precise rate coefficients for the collisional excitation of CO. Neufeld et al. presented cooling rates not only for the $\mathrm{CO}$ molecule but also for other major coolants like $\mathrm{H}_{2}, \mathrm{H}_{2} \mathrm{O}, \mathrm{O}_{2}, \mathrm{C}$ and $\mathrm{O}$, the steady state abundances of which were calculated using a complex chemical network. Both Goldsmith \& Langer and Neufeld et al. used a homogeneous cloud model and an escape probability formalism to obtain the cooling rates. A model which accounts for an inhomogeneous density and velocity structure was presented by Juvela et al. (2001). They obtained cooling rates for the major coolants by solving the full NLTE radiative transfer problem with Monte
Carlo methods for three different cloud models. The spatial variations of the density and velocity they used in each model cloud were a result of magnetohydrodynamic simulations.

In the present paper, we reexamine the influence of an inhomogeneous clumpy density distribution and of a turbulent velocity field on the cooling efficiency of molecular line emission considering $\mathrm{CO}$ as an example. We describe both the turbulent velocity field and the density structure by their statistical properties. Compared to other solution of the radiative transfer problem for a deterministic density and velocity structure, for example by means of a Monte Carlo method, a stochastic model has the advantage of an excellent computational performance, and, hence, a greater range of cloud parameters can be studied. According to previous work on NLTE line formation (cf. Hegmann \& Kegel 2000), we assume the logarithmic density spectrum and the turbulent velocity distribution to be Gaussians with exponential spatial correlation functions. This is consistent with the assumption that along each line of sight the variation of the logarithmic density and the turbulent velocity is governed by a Gauss Markov process. In this stochastic radiative transfer model (in the following referred to as SRTM), the velocity field and the density distribution are each characterized by two parameters: the mean quadratic turbulence velocity $\sigma_{\mathrm{v}}$, the standard deviation of the logarithmic density $\sigma_{\mathrm{n}}$, and the correlation lengths $\ell_{\mathrm{v}}$ and $\ell_{\mathrm{n}}$. As a consequence of the stochastic nature of the underlying density and velocity structure, the conventional radiative transfer equation must be replaced by a multidimensional generalized radiative transfer equation of Focker Planck type. 
The present paper has two principal objectives: At first, we examine the influence of stochastic density fluctuations and a turbulent velocity field with finite correlation length on the cooling efficiency of $\mathrm{CO}$ for a cold $\left(T_{\text {gas }}=(10-100) \mathrm{K}\right)$ and dense molecular medium $\left(n_{\mathrm{H}_{2}}=\left(10^{2}-10^{6}\right) \mathrm{cm}^{-3}\right)$ for a wide range of stochastic parameters $\sigma_{\mathrm{v}}, \sigma_{\mathrm{n}}, \ell_{\mathrm{v}}$, and $\ell_{\mathrm{n}}$. Secondly, we compare the SRTM results with the results of the other authors mentioned above. Since the approximations made in the frame of the SRTM imply some simplifications, we are in particular interested in whether the SRTM is able to reproduce the cooling rates of Juvela and collaborators who used cloud structures which were obtained by magnetohydrodynamic simulations.

\section{The stochastic radiative transfer model}

\subsection{Stochastic velocity field}

In this section, we shortly outline the basics of the stochastic radiative transfer model. For an in-depth discussion we refer to Hegmann \& Kegel (2000) and references therein. We describe the turbulent velocity field in terms of its stochastic properties. We consider the one-point distribution of $v$ (the turbulent velocity component along the line of sight) to be a Gaussian

$W_{\mathrm{v}}(v)=\frac{1}{\sqrt{2 \pi} \sigma_{\mathrm{v}}} \exp \left(-\frac{v^{2}}{2 \sigma_{\mathrm{v}}^{2}}\right)$,

where $\sigma_{\mathrm{v}}^{2}$ is the mean quadratic turbulent velocity. In addition, the variation of $v$ along each line of sight is assumed to be governed by a Markov process with an exponential two-point correlation function

$f(\Delta s)=\frac{\langle v(s) v(s+\Delta s)\rangle}{\sigma_{\mathrm{v}}^{2}}=\exp \left(-\frac{|\Delta s|}{\ell_{\mathrm{v}}}\right)$,

with $\ell_{\mathrm{v}}$ being the correlation length of the turbulent velocity field.

Due to the stochastic nature of the underlying velocity field the intensity becomes a stochastic variable, too. It can be shown that Eqs. (1) and (2) together with the ordinary radiative transfer equation

$\frac{\mathrm{d} I_{v}}{\mathrm{~d} s}=-\kappa_{v}\left(I_{v}-S_{v}\right)$

results in a Focker-Planck equation for the conditional intensity $q_{v}(v)$ (see Gail et al. 1974):

$$
\begin{aligned}
\frac{\partial q_{v}}{\partial s}= & \frac{1}{\ell_{\mathrm{v}}}\left(-v \frac{\partial q_{v}}{\partial v}+\sigma^{2} \frac{\partial^{2} q_{v}}{\partial v^{2}}\right) \\
& -\kappa_{v}\left(q_{v}-S_{v}\right) .
\end{aligned}
$$

The actual expectation value of the intensity can be calculated from $q_{v}(v)$ by

$$
\left\langle I_{v}\right\rangle=\int q_{v}(v) W_{\mathrm{v}}(v) \mathrm{d} v \text {. }
$$

The source function $S_{v}(v, s)$ and the absorption coefficient $\kappa_{v}(v, s)$ are given in the usual way by the occupation numbers $n_{i}$ and $n_{j}$ corresponding to the particular transition considered. This means, that in the case of NLTE, the generalized transfer equation has to be solved consistently with the set of rate equations (for a detailed derivation see Gail et al. 1975):

$$
\begin{aligned}
& \sum_{j \neq i}\left(n_{j}(v)\left(A_{i j}+C_{i j}\right)-n_{i}(v)\left(A_{j i}+C_{j i}\right)\right) \\
& +\sum_{j \neq i}\left(\frac{4 \pi}{c}\left\langle\widetilde{J}_{i j}(v)\right\rangle\left(n_{j}(v) B_{i j}-n_{i}(v) B_{j i}\right)\right)=0 .
\end{aligned}
$$

$A_{i j}$ and $B_{i j}$ are the Einstein coefficients for spontaneous and induced transitions $j \rightarrow i$, while $C_{i j}$ denotes the probability for collisional transitions. We calculated the Einstein coefficients for the $\mathrm{CO}$ molecule on the basis of a rigid rotor model using a dipole moment of $\mu=0.112$ Debye as given by Spitzer (1985). The collisional rate coefficients $C_{i j}$ were taken from the Leiden Atomic and Molecular Database (Schöier et al. 2005; Flower 2001). For the solid angle average of the intensity, we use the following approximative term introduced by Albrecht \& Kegel (1987):

$\left\langle\widetilde{J}_{i j}(v)\right\rangle \approx \frac{1}{4 \pi} \iint\left[q_{v}(v)\right]_{i j} \Phi(v, v) \mathrm{d} v \mathrm{~d} \Omega$,

where $\Phi(v, v)$ is the normalized profile of the local absorption coefficient under the assumption of complete redistribution over this local profile. The rate Eqs. (6) have to be solved for a number of energy levels sufficient to ensure that all significantly populated levels have been considered. Within the temperature range considered $(T \leq 100 \mathrm{~K})$, a maximum number of 18 energy levels is sufficient.

In this context, one may also ask for the power spectrum of the stochastic velocity fluctuations defined by Eqs. (1) and (2). Since the power spectrum is given by the Fourier transform of the autocorrelation function, Eq. (2) implies the following 1D and $3 \mathrm{D}$ power spectra:

$P_{1}(k) \propto \frac{\ell_{\mathrm{v}}}{1+\ell_{\mathrm{v}}^{2} k^{2}}$

and

$P_{3}(k) \propto \frac{\ell_{\mathrm{v}}}{\left(1+\ell_{\mathrm{v}}^{2} k^{2}\right)^{2}}$.

For $\left(\ell_{\mathrm{v}} k\right)^{2} \gg 1$, Eq. (9) can be approximated by a power law with spectral index $\beta=4$. A pure power law for scales within a certain interval, which would be expected for a self-similar turbulent energy cascade, seems to be consistent with oberservational data (see e.g. Sun et al. 2006; Ossenkopf et al. 2006).

\subsection{Stochastic density fluctuations}

Equation (4) which accounts for a stochastic velocity field is only valid for a given deterministic density distribution. Since we are also interested in the effects of a clumpy density distribution on the molecular cooling rates, a stochastic description of the density variation similar to the turbulent velocity case is given here (cf. Traving 1980 and Hegmann \& Kegel 2000). At first, it is convenient to transform the molecular hydrogen number density $n_{\mathrm{H}_{2}}$ to a logarithmic scale by introducing a logarithmic density $\tilde{n}$ :

$\tilde{n} \stackrel{\text { def }}{=} \ln \left(\frac{n_{\mathrm{H}_{2}}}{n_{\text {ref }}}\right)$.

with $n_{\text {ref }}$ being the reference density. Analogues to Eqs. (1) and (2), the variation of the logarithmic density is assumed to be given by a Gauss Markov process. The one-point distribution of $\tilde{n}$ and the correlation function are given by:

$$
\begin{aligned}
& W_{\mathrm{n}}(v)=\frac{1}{\sqrt{2 \pi} \sigma_{\mathrm{n}}} \exp \left(-\frac{\tilde{n}^{2}}{2 \sigma_{\mathrm{n}}^{2}}\right) \\
& g(\Delta s)=\frac{\langle\tilde{n}(s) \tilde{n}(s+\Delta s)\rangle}{\sigma_{\mathrm{n}}^{2}}=\exp \left(-\frac{|\Delta s|}{\ell_{\mathrm{n}}}\right)
\end{aligned}
$$


where $\sigma_{\mathrm{n}}$ and $\ell_{\mathrm{n}}$ are the standard deviation of the logarithmic density distribution and the correlation length the density field. The generalized radiative transfer equation which follows from Eqs. (11) and (12) together with the ordinary radiative transfer equation is given by

$$
\begin{aligned}
\frac{\partial q_{v}}{\partial s}= & \frac{1}{\ell_{\mathrm{n}}}\left(-v \frac{\partial q_{v}}{\partial \tilde{n}}+\sigma_{\mathrm{n}}^{2} \frac{\partial^{2} q_{v}}{\partial \tilde{n}^{2}}\right) \\
& -\kappa_{v}\left(q_{v}-S_{v}\right) .
\end{aligned}
$$

The implications of the assumptions made in Eqs. (10)-(12) were discussed by Hegmann \& Kegel (2000). It is shown that $\sigma_{\mathrm{n}}$ is a measure to which extent the mass of the cloud is concentrated in dense clumps, whereas the correlation length $\ell_{\mathrm{n}}$ is a length scale for the variation of the density. The clumps become denser but smaller with increasing $\sigma_{\mathrm{n}}$. In writing Eq. (13) we neglected the correlations of the velocity field. This means that the turbulent velocity enters the radiative transport in the usual microturbulent limit only. A form of the generalized radiative transfer equation which accounts for both density and velocity fluctuations was given by Hegmann \& Kegel (2000):

$$
\begin{aligned}
\frac{\partial q_{v, \mathbf{s}}}{\partial s}= & \frac{1}{\ell_{\mathrm{n}}}\left(-\tilde{n} \frac{\partial q_{v, \mathbf{s}}}{\partial \tilde{n}}+\sigma_{\mathrm{n}}^{2} \frac{\partial^{2} q_{v, \mathbf{s}}}{\partial \tilde{n}^{2}}\right)+\frac{1}{\ell_{\mathrm{v}}} \frac{\partial}{\partial v}\left(-v \frac{\partial q_{v, \mathbf{s}}}{\partial v}+\sigma_{\mathrm{v}}^{2} \frac{\partial^{2} q_{v, \mathbf{s}}}{\partial v^{2}}\right) \\
& -\kappa_{v}\left(q_{v, \mathbf{s}}-S_{v}\right) .
\end{aligned}
$$

However, in the present paper, we focus on the seperate study of the influence of stochastic velocity and density fluctuations by using Eqs. (4) and (13). This allows to identify clearly the individual contribution of each of these stochastic fluctuations to the cooling mechanism. Moreover, combining both effects would not only increase the number of independent variables (from 2 to 3 ) and the number of free parameters (from 2 to 4 ), but also the necessary computing time.

\section{The cloud model}

To study the different effects of density and velocity fluctuations on the $\mathrm{CO}$ cooling rates, we perform numerical simulations based on three different cloud models. In the first model A, we assume a homogeneous sphere with a turbulent velocity field with finite correlation length. In model B, we allow for a clumpy stochastically distributed density. However, the turbulent velocity field in model B is described in the usual microturbulent approximation only. And finally in model C, both, velocity and density fluctuations are accounted for. The clouds are embedded in the cosmic background radiation in all three cases.

The radiation field for the different rotational transitions of $\mathrm{CO}$ is calculated for each cloud model by solving the coupled Eqs. (4), (13) or (13), (6), and (7) numerically. The gas temperature and the relative $\mathrm{CO}$ abundance are considered to be constant throughout the cloud. For the latter, we adopt a value $n_{\mathrm{CO}} / n_{\mathrm{H}_{2}}=8 \times 10^{-5}$ as given by Black \& Willner (1984), which is somewhat smaller than predicted by standard chemical models (e.g. Millar et al. 1997). We will consider average molecular hydrogen number densities between $n_{\mathrm{H}_{2}}=10^{2} \mathrm{~cm}^{-3}$ and $n_{\mathrm{H}_{2}}=10^{6} \mathrm{~cm}^{-3}$ and gas temperatures between $T_{\text {gas }}=10 \mathrm{~K}$ and $T_{\text {gas }}=100 \mathrm{~K}$.

\section{Results}

\subsection{Turbulent velocity field with finite correlation length}

The CO cooling rates $\Lambda$ are mainly a function of three parameters: the density, the kinetic gas temperature, and the local radiation field. Whereas the first two are assumed to be constant

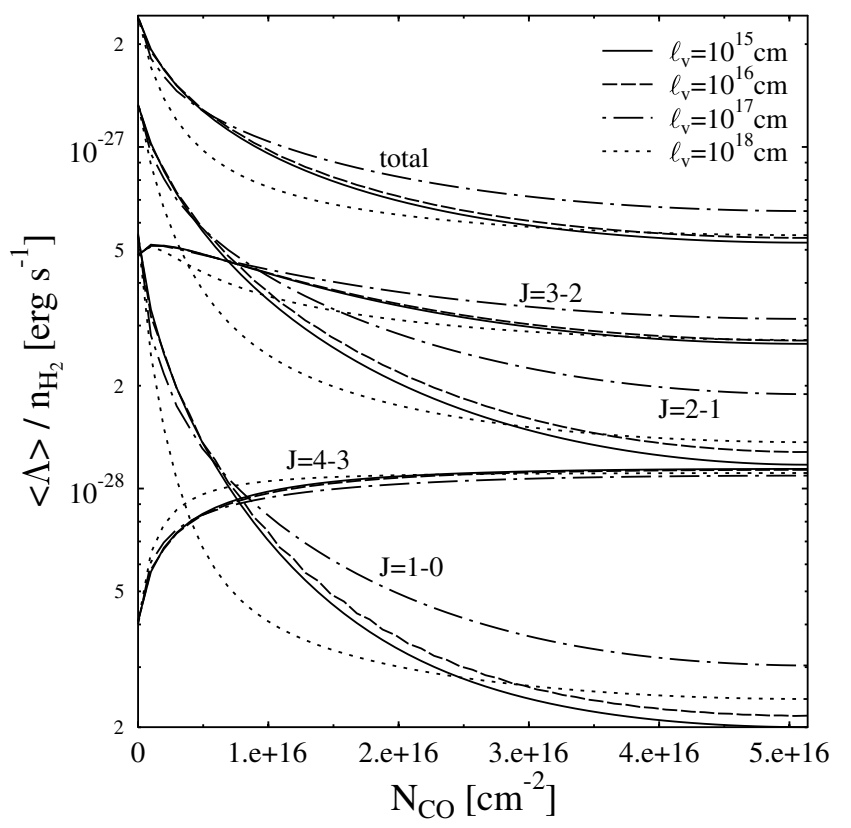

Fig. 1. Selected CO cooling rates $\langle\Lambda\rangle / n_{\mathrm{H}_{2}}$ as a function of the CO column density for a single cloud (model A) with $T_{\text {gas }}=10 \mathrm{~K}, \sigma_{\mathrm{v}}=$ $0.2 \mathrm{~km} \mathrm{~s}^{-1}, n_{\mathrm{H}_{2}}=500 \mathrm{~cm}^{-3}$, and $\mathcal{M}=10 \mathcal{M}_{\text {sun }}$.

in this section, the latter depends on the spatial coordinate. In general, the mean intensity in the CO lines increases with increasing optical depth and converges slowly to the Planck function $B_{v}\left(T_{\text {gas }}\right)$. For a single cloud with $\mathcal{M}=10 \mathcal{M}_{\text {sun }}, T_{\text {gas }}=10 \mathrm{~K}$, $n_{\mathrm{H}_{2}}=500 \mathrm{~cm}^{-3}, \sigma_{\mathrm{v}}=0.2 \mathrm{~km} \mathrm{~s}^{-1}$, and four different correlation lengths, the velocity averaged $\mathrm{CO}$ cooling rates normalized to the hydrogen number density are given as a function of the CO column density (measured from the cloud's edge to the center) in Fig. 1. It can be clearly seen, that the cooling rates of the lowest rotational transitions $J=1 \rightarrow 0$ and $J=2 \rightarrow 1$ decline steeply towards the cloud center, whereas cooling by the high rotational transition $J=4 \rightarrow 3$ increases at the same time. With increasing optical depth, the escape probability for the photons decreases and the cooling efficiency of the lower rotational transitions drops. On the other hand, with an increased line intensity towards the cloud center the occupation number of the upper level of the respective transition rises. As a consequence, the probability to excite still higher rotational states by $\mathrm{CO}-\mathrm{H}_{2}$ collisions is clearly larger at the cloud center than at the cloud's edge. So, for example, the collisional rate coefficient for the $J=2 \rightarrow 4$ transition at $T_{\text {gas }}=10 \mathrm{~K}$ is about 20 times larger than for the $J=0 \rightarrow 4$ transition. Due to this effect, the cooling rates of the high rotational lines become larger with an increasing optical depth of the low rotational lines. The influence of the correlation length of the turbulent velocity field $\ell_{\mathrm{v}}$ on the cooling efficiency can be seen best for the optically thick $J=1 \rightarrow 0$ and $J=2 \rightarrow 1$ lines. In the optically thin limit, the correlation length has no influence on the cooling rates at all. Hence, the graphs for different $\ell_{\mathrm{v}}$ converge at $N_{\mathrm{CO}}=0$. For moderately large CO column densities, the effective absorption coefficient of the turbulent medium increases as the ratio of the correlation length to the mean free path of photons decreases (cf. Albrecht $\&$ Kegel 1987 and Kegel et al. 1993). Consequently, the outer layers of the cloud become optically more opaque with decreasing $\ell_{\mathrm{v}}$. This can be seen in Fig. 1 where the cooling rate for the $J=1 \rightarrow 0$ and $J=2 \rightarrow 1$ lines are larger for the smaller correlation length at the cloud's edge. In contrast, the cooling 

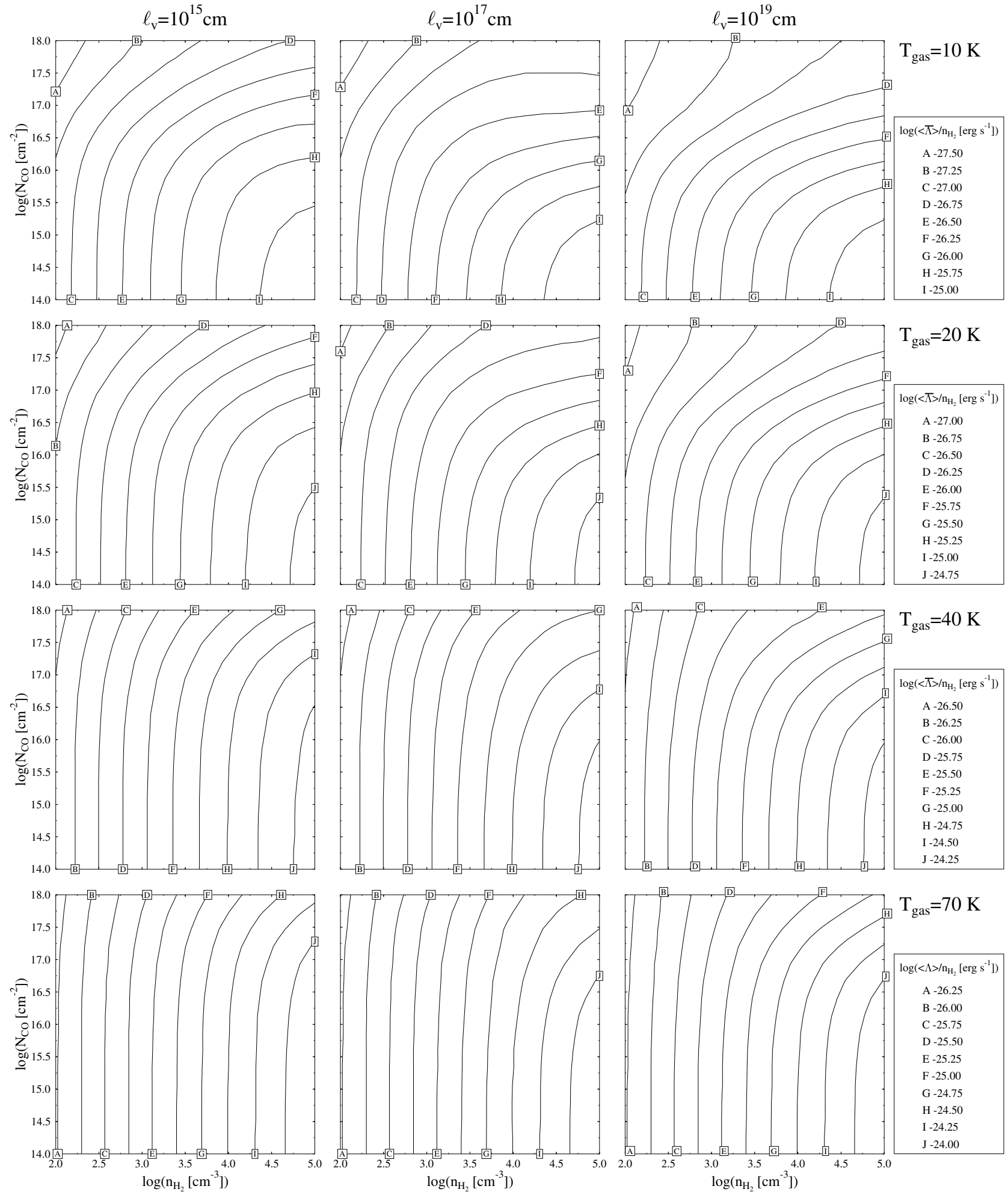

Fig. 2. Volume and velocity averaged CO cooling rates for a large set of clouds (model A) with different masses and densities. The cooling rate is given as a function of the cloud's density and the $\mathrm{CO}$ column density along the cetral line of sight. From the left to the right, the correlation length of the turbulent velocity field is increased. For $T_{\text {gas }}=(10,20) \mathrm{K}$, a turbulent velocity of $\sigma_{\mathrm{v}}=0.2 \mathrm{~km} \mathrm{~s}^{-1}$ is assumed, while $\sigma_{\mathrm{v}}=0.5 \mathrm{~km} \mathrm{~s}{ }^{-1}$ for $T_{\text {gas }}=(40,70) \mathrm{K}$.

rate becomes minimal for the microtutbulent case $\ell_{\mathrm{v}} \rightarrow 0$ in the cloud center. Here, photons escape hardly from the cloud for a small $\ell_{\mathrm{v}}$. Along every line of sight there is almost certainly a turbulent element with the appropriate radial velocity to reabsorb the emitted photon.
Figure 2 summarizes the spatially averaged $\mathrm{CO}$ cooling rates for a large set of clouds with varying mass and density. The total, volume averaged cooling rates of the clouds are given as a function of their hydrogen number densities and the CO column densities along their central lines of sight. In each single frame, 


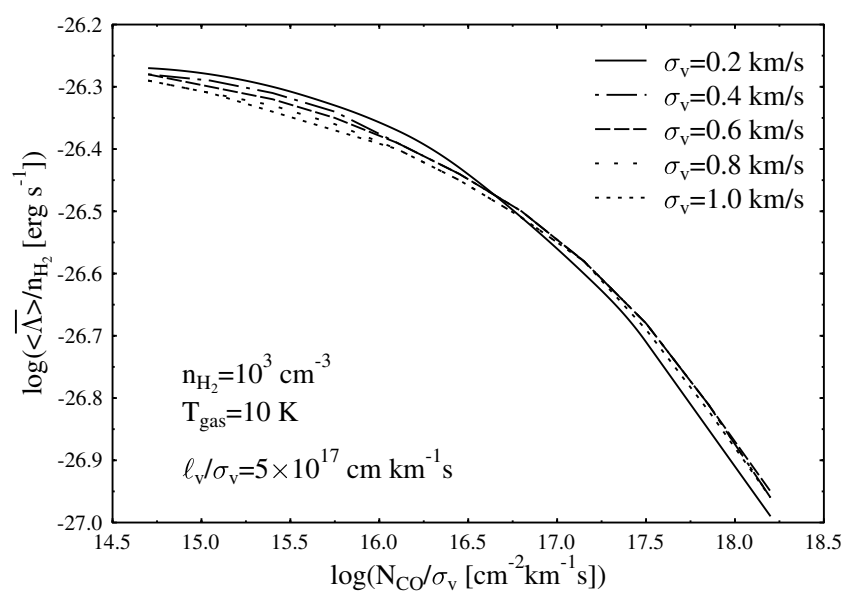

Fig. 3. Volume and density averaged cooling rates $\langle\bar{\Lambda}\rangle / n_{\mathrm{H}_{2}}$ for a set of clouds with the same density but different masses. The cooling rates are given as a function of the normalized $\mathrm{CO}$ colum density along the central lines of sight of each cloud.

the temperature, the mean quadratic turbulent velocity, and the correlation length of the turbulent velocity field of the clouds are kept constant, whereas from one frame to the other the correlation length and the gas temperature are altered. For the assumed $\mathrm{CO} / \mathrm{H}_{2}$ ratio $n_{\mathrm{CO}} / n_{\mathrm{H}_{2}}=8 \times 10^{-5}$, the masses of the clouds can be obtained at each point in the contour plot by

$\mathcal{M}=\frac{1}{6} \pi\left(\frac{N_{\mathrm{CO}}}{n_{\mathrm{CO}}}\right)^{3} 1.2 n_{\mathrm{H}_{2}} \mu m_{\mathrm{u}}$,

where the factor 1.2 accounts for a $10 \%$ ratio of $\mathrm{He}$ to $\mathrm{H}$ atoms, $\mu$ is the mean molecular weight and $m_{\mathrm{u}}$ is the atomic mass unit. For a small CO column density, all rotational lines of CO are optically thin. At a certain critical column density $N_{\text {crit }}$, the dominant cooling lines become optically thick and the overall cooling rate starts to drop. Due to strong deviations from LTE, $N_{\text {crit }}$ depends on the local density: the larger $n_{\mathrm{H}_{2}}$ is, the earlier the decrease of the cooling efficiency sets in. The critical column density $N_{\text {crit }}$ is also a function of the temperature and becomes larger with increasing temperature. For the high temperature $T_{\text {gas }}=70 \mathrm{~K}$, more, and consequently optically thinner rotational transitions of $\mathrm{CO}$ dominate the cooling rate than in the $T_{\text {gas }}=10 \mathrm{~K}$ case. The influence of the correlation length on the cooling rates becomes significant for large $n_{\mathrm{H}_{2}}$ and $N_{\mathrm{CO}}$. Except for rather low hydrogen number densities, $n_{\mathrm{H}_{2}}<10^{3} \mathrm{~cm}^{-3}$, and high column densities, the cooling efficiency is largest for the microturbulent case $\ell_{\mathrm{v}} \rightarrow 0$ and decreases with increasing $\ell_{\mathrm{v}}$. However, this is true for the spatially averaged cooling rate only. Locally, the dependence of the cooling rate with the correlation length may reverse (cf. Fig. 1).

Up to now, the width $\sigma_{\mathrm{v}}$ of the turbulent velocity distribution was kept constant at $0.2 \mathrm{~km} \mathrm{~s}^{-1}$ and $0.5 \mathrm{~km} \mathrm{~s}^{-1}$, respectively, in all calculations. To study the influence of $\sigma_{\mathrm{v}}$ on the cooling efficiency it is convenient to replace the independent variables $N_{\mathrm{CO}}$ and $\ell_{\mathrm{v}}$ by their normalized counterparts $N_{\mathrm{CO}} / \sigma_{\mathrm{v}}$ and $\ell_{\mathrm{v}} / \sigma_{\mathrm{v}}$. In Fig. 3, a set of curves for $\sigma_{\mathrm{v}}=0.2 \ldots 1.0 \mathrm{~km} \mathrm{~s}^{-1}$ and a constant $\ell_{\mathrm{v}} / \sigma_{\mathrm{v}}$ is given as a function of $N_{\mathrm{CO}} / \sigma_{\mathrm{v}}$. The cooling rates changes only slightly with $\sigma_{\mathrm{v}}$, whereas the greatest changes are obtained for small $\sigma_{\mathrm{v}}$ and small $N_{\mathrm{CO}} / \sigma_{\mathrm{v}}$. The deviations can be described, at least in part, by the thermal contribution to the total velocity of the molecule which increases with decreasing $\sigma_{\mathrm{v}}$. For $\sigma_{\mathrm{v}}=0.2 \mathrm{~km} \mathrm{~s}^{-1}$, the ratio of the mean turbulent velocity to

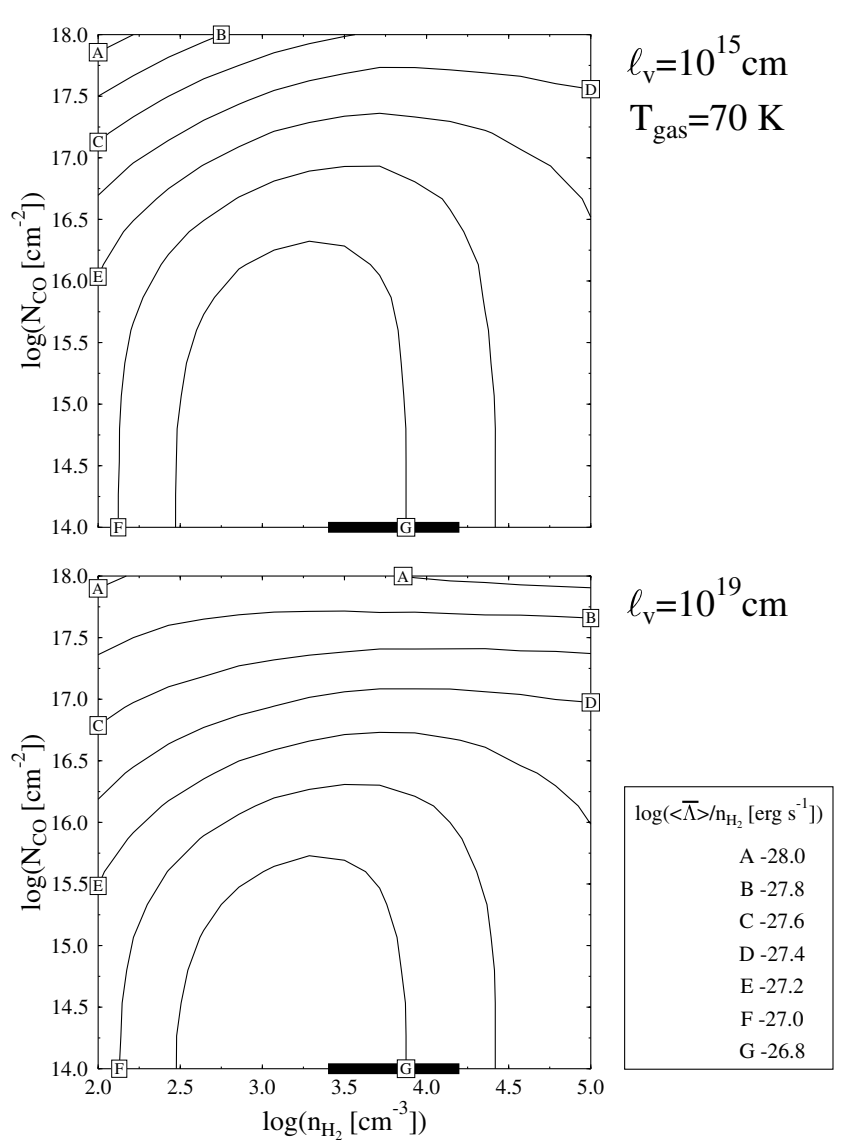

Fig. 4. Volume and velocity averaged $\mathrm{CO}$ cooling rates for the $J=1 \rightarrow$ 0 transition as a function of the cloud's density and the $\mathrm{CO}$ column density along the cetral line of sight (model A). The turbulent velocity is $\sigma_{\mathrm{v}}=0.4 \mathrm{~km} \mathrm{~s}^{-1}$ and the gas temperature is $T_{\text {gas }}=70 \mathrm{~K}$. The range of critical densities for which an inversion of the $J=1 \rightarrow 0$ line shows up is marked on the $x$-axis.

the thermal velocity takes a value of only $\sigma_{\mathrm{v}} / v_{\mathrm{th}}=2.6$, whereas for $\sigma_{\mathrm{v}}=1.0 \mathrm{~km} \mathrm{~s}^{-1}$ this ratio is 10.5 .

\subsection{Population inversion}

With increasing gas temperature, initially suprathermal excitations and eventually population inversions in the low rotational levels of $\mathrm{CO}$ can be found. According to Köppen \& Kegel (1980), an inversion for the $J=1 \rightarrow 0$ line arises for $T_{\text {gas }} \geq 50 \mathrm{~K}$ and $\mathrm{H}_{2}$ densities about $10^{4} \mathrm{~cm}^{-3}$. The inversion is due to the excitation of high rotational levels of $\mathrm{CO}$ by collisions with $\mathrm{H}_{2}$ and the spontaneous decay rate increasing with increasing angular momentum $J$, causing a "traffic jam" in the lower levels. Since an inversion of the $J=1 \rightarrow 0$ transition is only present for small CO column densities, the masers Köppen \& Kegel found on the basis of their on-the-spot model were optically thin in all cases. This is also true for our stochastic radiative transfer model. However, since single turbulence elements can decouple from the rest of the cloud due to the Doppler effect for a large turbulent velocity component, $\mathrm{CO}$ maser spots can be found within the cloud for much larger $\mathrm{CO}$ column densities than in the case of a homogeneous medium. We found negative excitation temperatures for CO column densities up to $N_{\mathrm{CO}}=10^{18}$. Of course, the spatial grid must be fine enough to resolve single maser spots, and consequently the computing time steeply increases with increasing radius of the cloud. In Fig. 4, the cooling rates 


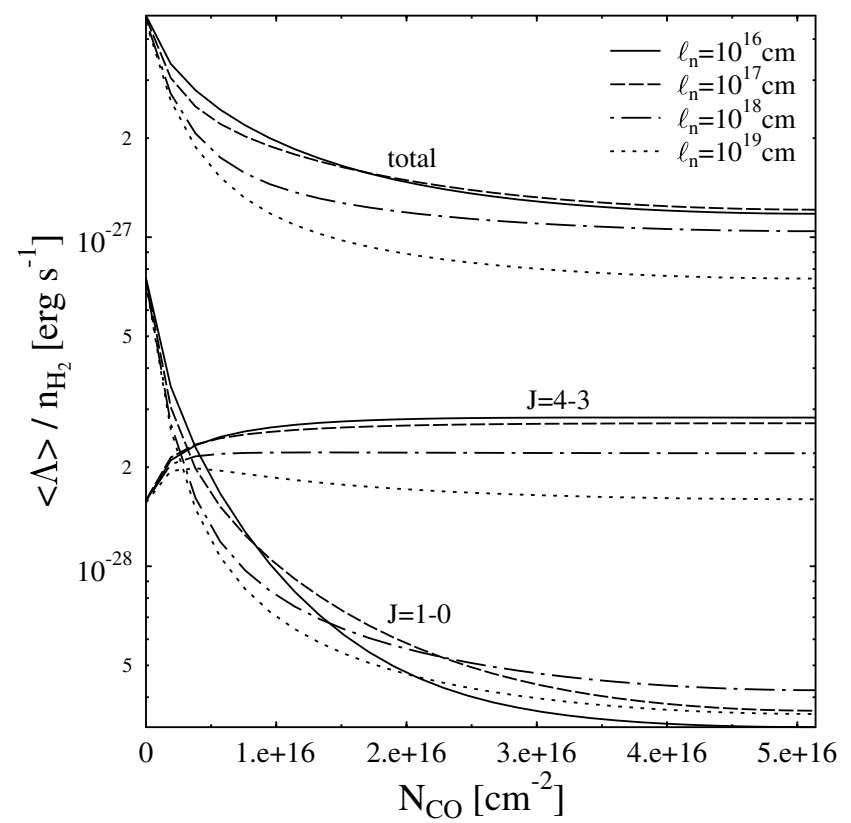

Fig. 5. Selected $\mathrm{CO}$ cooling rates $\langle\Lambda\rangle / n_{\mathrm{H}_{2}}$ as a function of the CO column density for an inhomogeneous microturbulent cloud model (model B) with $T_{\text {gas }}=10 \mathrm{~K}, \sigma_{\mathrm{v}}=0.2 \mathrm{~km} \mathrm{~s}^{-1},\left\langle n_{\mathrm{H}_{2}}\right\rangle=500 \mathrm{~cm}^{-3}$, $\sigma_{\mathrm{n}}=1.0$ and $\mathcal{M}=10 \mathcal{M}_{\text {sun }}$.

for the $J=1 \rightarrow 0$ transition are shown as a function of the cloud radius and the $\mathrm{H}_{2}$ density for $T_{\text {gas }}=70 \mathrm{~K}$. The critical range of $\mathrm{H}_{2}$ densities for which an inversion of the $J=1 \rightarrow 0$ line shows up is marked on the $\mathrm{x}$-axis.

\subsection{Stochastic density fluctuations}

In this section, we investigate how the cooling by $\mathrm{CO}$ is affected by an inhomogeneous stochastic density distribution. For this, we use model B which accounts for density fluctuations but describes the turbulent Doppler broadening by a conservative microturbulent approximation which implies complete redistribution of photons over the whole line profile (thermal + turbulent). This is in cotrast to model A, where only complete redistribution over the thermal profile is assumed, while the redistribution in frequency caused by the turbulent velocity is partial (see Kegel et al. 1993). Figures 5 and 6 show the local cooling rates for two different rotational transitions as a function of the distance from the cloud edge for a cloud with $\mathcal{M}=10 \mathcal{M}_{\text {sun }}, T_{\text {gas }}=10 \mathrm{~K}$, and $\left\langle n_{\mathrm{H}_{2}}\right\rangle=500 \mathrm{~cm}^{-3}$. Whereas $\sigma_{\mathrm{n}}$ is kept constant and $\ell_{\mathrm{n}}$ is varied in Fig. 5, in Fig. 6, $\sigma_{\mathrm{n}}$ adopts four different values and $\ell_{\mathrm{n}}$ is kept constant. The density fluctuations act upon the cooling rates by essentially two mechanisms. On the one hand, radiative transfer itself is affected. To describe the optical properties of a medium with stochastic density fluctuations an effective absorption coefficient $\kappa_{\text {eff }}$ can be introduced, if the occupation numbers are given by a Boltzmann distribution. As has been shown by Hegmann \& Kegel (2003), $\kappa_{\text {eff }}$ decreases with both, an increasing standard deviation of the logarithmic density distribution $\sigma_{\mathrm{n}}$ and increasing correlation length $\ell_{\mathrm{n}}$. As a result, the local cooling rates are - compared to the homogeneous case - decreased at the cloud's edge and increased in the cloud center for strong density fluctuations with a large correlation length. This effect can be clearly seen for the transition $J=1 \rightarrow 0$ in Figs. 5 and 6 which is effected much less by NLTE effects than the higher rotational transitions.

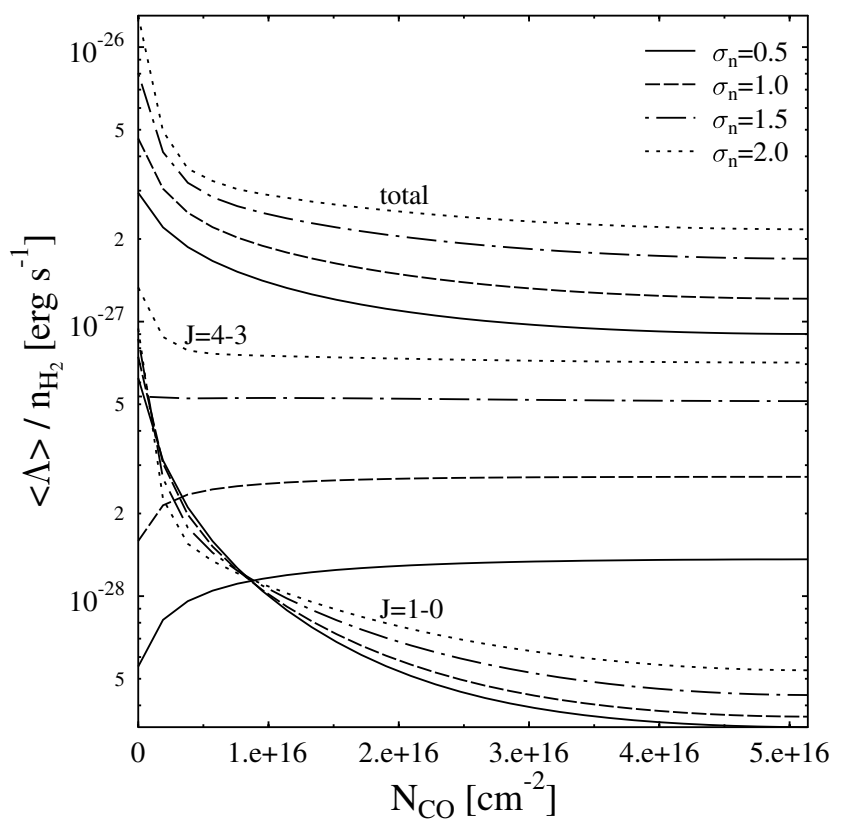

Fig. 6. Selected $\mathrm{CO}$ cooling rates $\langle\Lambda\rangle / n_{\mathrm{H}_{2}}$ as a function of the $\mathrm{CO}$ column density for an inhomogeneous microturbulent cloud model (model B) with $T_{\text {gas }}=10 \mathrm{~K}, \sigma_{\mathrm{v}}=0.2 \mathrm{~km} \mathrm{~s}^{-1},\left\langle n_{\mathrm{H}_{2}}\right\rangle=500 \mathrm{~cm}^{-3}$, $\ell_{\mathrm{n}}=10^{17} \mathrm{~cm}$ and $\mathcal{M}=10 \mathcal{M}_{\text {sun }}$.

On the other hand, density fluctuations affect directly the local occupation numbers of the $\mathrm{CO}$ molecule. Since the transition rates due to $\mathrm{CO}-\mathrm{H}_{2}$ collisions depend on the square of the density, the mean collisional rates depend not only on the mean density but also on the second moment $\sigma_{\mathrm{n}}^{2}$ of the logarithmic density distribution. As a result, the mean collisional rates are strongly enhanced for large values of $\sigma_{\mathrm{n}}$. The influence on the cooling rates by this effect can be seen best for the high $J=4 \rightarrow 3$ transition in Fig. 6: although the mean density stays constant, the average cooling rate is dramatically increased with $\sigma_{\mathrm{n}}$ becoming larger. In Fig. 7, the conditional expectation value $\lambda(\tilde{n}, s)$ of the local cooling rate is given as a function of the distance from the cloud's edge and the local density for $\sigma_{\mathrm{n}}=1.0$ and $\ell_{\mathrm{n}}=10^{16} \mathrm{~cm}$ $\left(10^{19} \mathrm{~cm}\right)$. The conditional expectation value $\lambda(\tilde{n}, s)$ denotes the expectation value of the cooling rate at point $s$ for a given logarithmic density $\tilde{n}$. The actual expectation value $\langle\Lambda(s)\rangle$ of the cooling rate can be calculated from $\lambda(\tilde{n}, s)$ by

$\langle\Lambda(s)\rangle=\int \lambda(\tilde{n}, s) W_{\mathrm{n}}(\tilde{n}) \mathrm{d} \tilde{n}$.

It can be seen, that $\lambda(\tilde{n}, s)$ increases strongly with an increasing density. This means, that most of the cooling power in inhomogeneous clouds comes from regions denser than the average. However, as the dense regions become optically opaque to most of the cooling lines, the increase of $\lambda(\tilde{n}, s)$ with the local density flattens. Since the size of the dense clumps scales with the correlation length $\ell_{\mathrm{n}}$, the flattening becomes pronounced for a large $\ell_{\mathrm{n}}$.

Figure 8 summarizes the spatially averaged $\mathrm{CO}$ cooling rates for a large number of cloud models with different mean hydrogen number densities and radii. $N_{\mathrm{CO}}$ denotes hereby the mean CO column densities along the central lines of sight. The other parameters $-\sigma_{\mathrm{v}}, \sigma_{\mathrm{n}}, \ell_{\mathrm{n}}, T_{\text {gas }}-$ are kept constant within each single frame. From the left to the right the Gaussian width of the logarithmic density distribution is increased form $\sigma_{\mathrm{n}}=0.5$ to $\sigma_{\mathrm{n}}=2.0$. It can be clearly seen that for a small mean hydrogen density the mean cooling rates are increased dramatically for an 

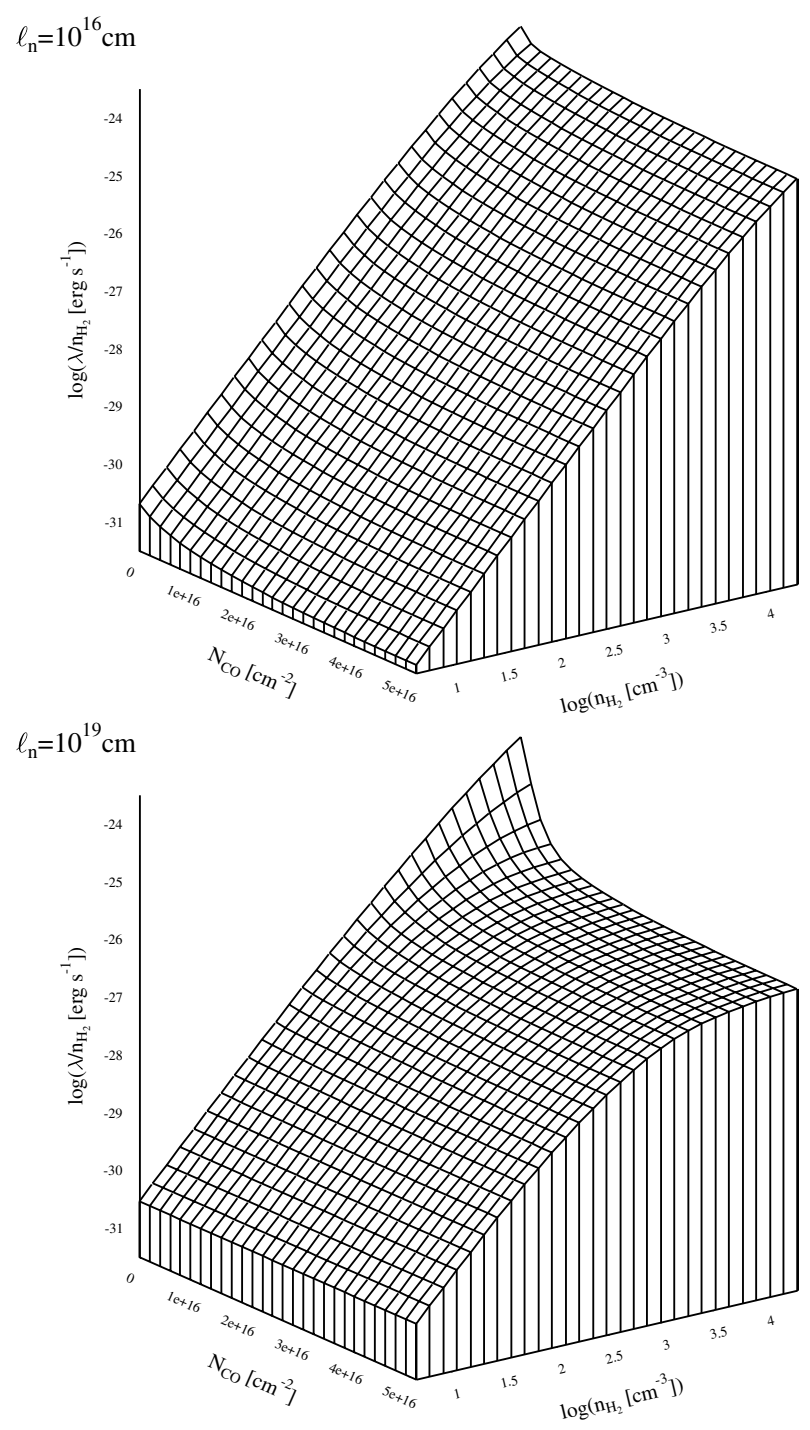

Fig. 7. Conditional expectation value of the local cooling rate as a function of the distance from the clooud's edge and the local density for an inhomogeneous microturbulent cloud model (model B) with $T_{\mathrm{gas}}=10 \mathrm{~K}, \sigma_{\mathrm{v}}=0.2 \mathrm{~km} \mathrm{~s}^{-1},\left\langle n_{\mathrm{H}_{2}}\right\rangle=500 \mathrm{~cm}^{-3}, \sigma_{\mathrm{n}}=1.0$ and $\mathcal{M}=10 \mathcal{M}_{\text {sun }}$.

increased strength of the density fluctuations. Most of the energy is radiated away from dense regions where the occupation numbers of the $\mathrm{CO}$ molecule are almost thermalized. However for a large value of $\left\langle n_{\mathrm{H}_{2}}\right\rangle$, the dependence of the cooling efficiency on $\sigma_{\mathrm{n}}$ reverses, because photon trapping reduces the cooling rates inside the dense clumps. Again, the effect becomes more pronounced for the larger correlation length.

\subsection{Combined density and velocity fluctuations}

In this section, the effects of combined density and velocity fluctuations are demonstrated on the basis of model $\mathrm{C}$ by solving Eq. (14). Figures $10 \mathrm{a}$ and $10 \mathrm{~b}$ give the total, volume, density and velocity averaged $\mathrm{CO}$ cooling rate for a series of clouds as a function of the $\mathrm{CO}$ column density along the central line of sight. Along each single curve, the cloud radius is increased, whereas the other physical paramters are kept constant. In Fig. 10a, the dependence of the cooling efficiency on the correlation length of the turbulent velocity field is examined. As in the case of pure velocity fluctuations (model A), an increasing correlation length tends to result in a decreasing cooling rate. Only for large $\mathrm{CO}$ column densities and $\mathrm{H}_{2}$ densites not larger than a few $10^{3} \mathrm{~cm}^{-3}$, the cooling efficiency has a maximum for a finite $\ell_{\mathrm{v}} \neq 0$. However, the dependence of the cooling efficiency is rather weak for this parameter regime. In Fig. 10b, the Gaussian width of the logarithmic density distribution is varied for otherwise constant parameters of the density and velocity field. The cooling rate is again increasing with increasing $\sigma_{\mathrm{n}}$, the largest differences occuring for small column densities.

\section{Comparison with earlier work}

In this section, the cooling rates obtained from the stochastic radiative transfer model are compared to earlier results by Neufeld et al. (1995) and Juvela et al. (2001). The cooling rates by Neufeld et al. are based on a large velocity gradient approximation and apply to clouds with a continuous and smooth density distribution. In particular, the density gradient has to be small on the scale length of the mean free path of photons. Their cooling rates are given as a function of a column density measure, i.e. the number density of $\mathrm{CO}$ divided by the velocity gradient: $\tilde{N}=G n_{\mathrm{CO}} /|\mathrm{d} v / \mathrm{d} x|$. The quantity $G$ is a dimensionless geometric scaling factor, which can be used to adapt the results of Neufeld et al. to different geometries. According to Neufeld \& Kaufmann (1993), $\tilde{N}$ can be approximated by $2 n_{\mathrm{CO}} r /(27 \Delta v)$ for a static sphere of radius $r$ and volume averaged cooling rates. We identify the velocity dispersion $\Delta v$ of the Neufeld model with the square root of the mean quadratic turbulent velocity $\sigma_{\mathrm{v}}^{2}$ of the stochastic radiative transfer model in the following. In Figs. 11 and 12 , the results obtained for model A (turbulent velocity field with finite correlation length and a homogeneous density distribution) are compared with the results as given by Neufeld and collaborators. In general, the cooling rates obtained from model A are smaller than the predictions from Neufeld et al., but the differences become much smaller with decreasing correlation length $\ell_{\mathrm{n}}$. The different cooling rates which exist at $T_{\text {gas }}=100 \mathrm{~K}$ even for an optically thin cloud can be explained by the different collisional rate coefficients used in both models (Flower \& Launay 1985; Flower 2001).

Juvela et al. (2001) computed CO cooling rates for inhomogeneous model clouds, the density and velocity structure of which are based on supersonic magnetohydrodynamic turbulence simulations by Padoa \& Nordlund (1999). In order to study the cooling rates for clouds with varying mean densities and temperatures, the original MHD data was scaled to different temperature and density regimes. In the following, we compare our model $\mathrm{C}$ results (combined density and velocity fluctuations) with the original unscaled cloud model. In accordance with our findings they concluded that an inhomogeneous density field reduces photon trapping. Further, Juvela et al. found that most of the cooling at low-to-intermediate densities is provided by clumps above the average gas density. In Fig. 13, the local cooling rates according to the model B of Juvela et al. (dotted line) are plotted as a function of the local density. In their model $\mathrm{B}$, the rms velocity is approximately $2.1 \mathrm{~km} \mathrm{~s}^{-1}$ at $T_{\text {gas }}=10 \mathrm{~K}$. The mean density and the linear size of the cubical cloud model are $\left\langle n_{\mathrm{H}_{2}}\right\rangle=320 \mathrm{~cm}^{-3}$ and $L=6.3 \mathrm{pc}$, respectively. It can be seen, that the cooling rate strongly increases with the local density at first. For very high densities, however, the curve flattens, because the dense clumps become optically opaque. In the same figure, the spatially and velocity averaged conditional expectation value of the local cooling rate $\langle\bar{\lambda}\rangle(n)$ for our model $\mathrm{C}$ (solid and dashed lines) is also shown. To give a comparison to the results of Juvela et al. (2001), the 

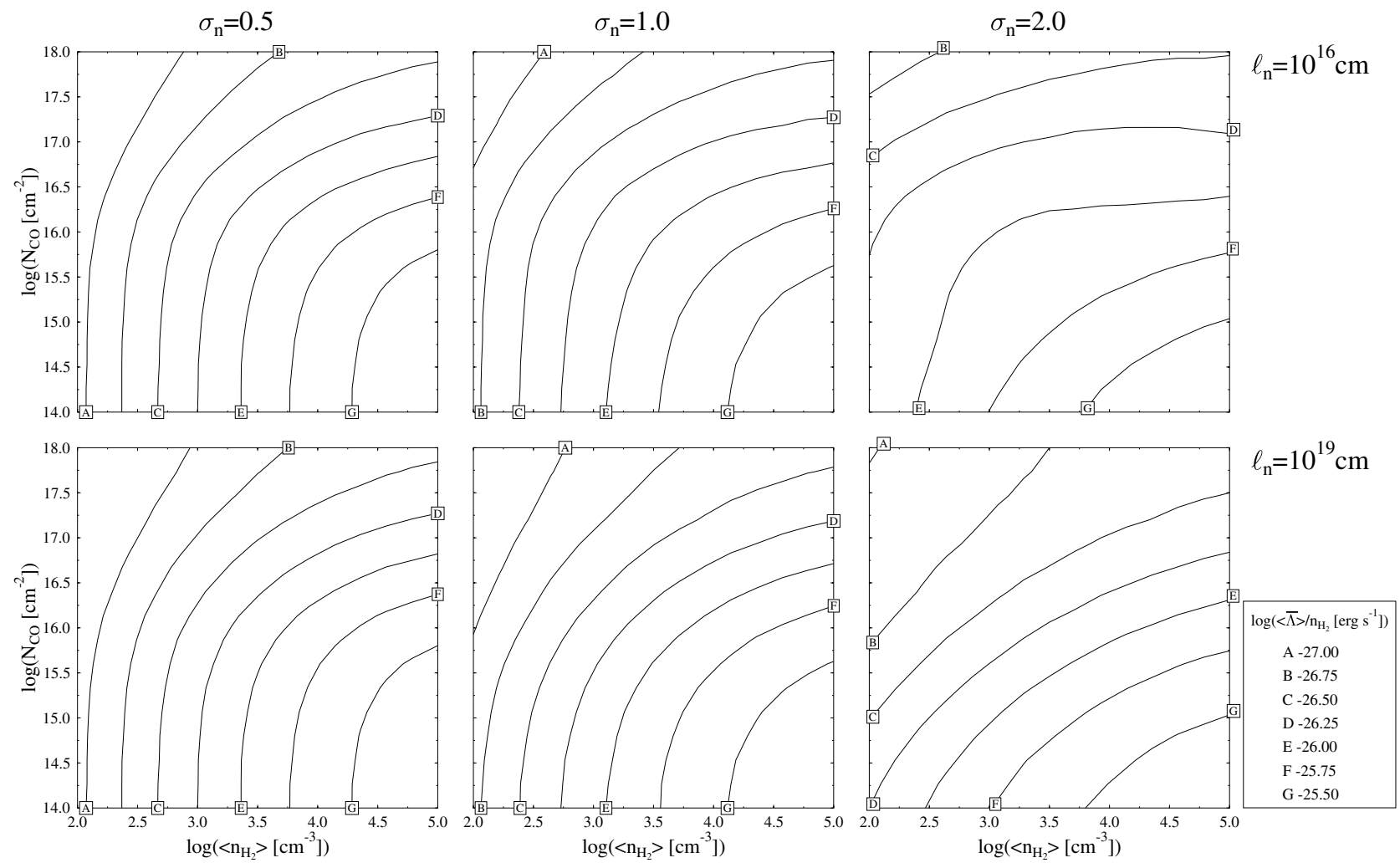

Fig. 8. Volume and density averaged CO cooling rates for a large set of clouds (model B) with different masses and densities. The cooling rate is given as a function of the mean cloud's density and the $\mathrm{CO}$ column density along the cetral line of sight. From the left to the right, $\sigma_{\mathrm{n}}$ is increased fro 0.5 to 2.0. The turbulent velocity is $\sigma_{\mathrm{v}}=0.2 \mathrm{~km} \mathrm{~s}^{-1}$ and the gas temperature is $T_{\text {gas }}=10 \mathrm{~K}$ in all models.
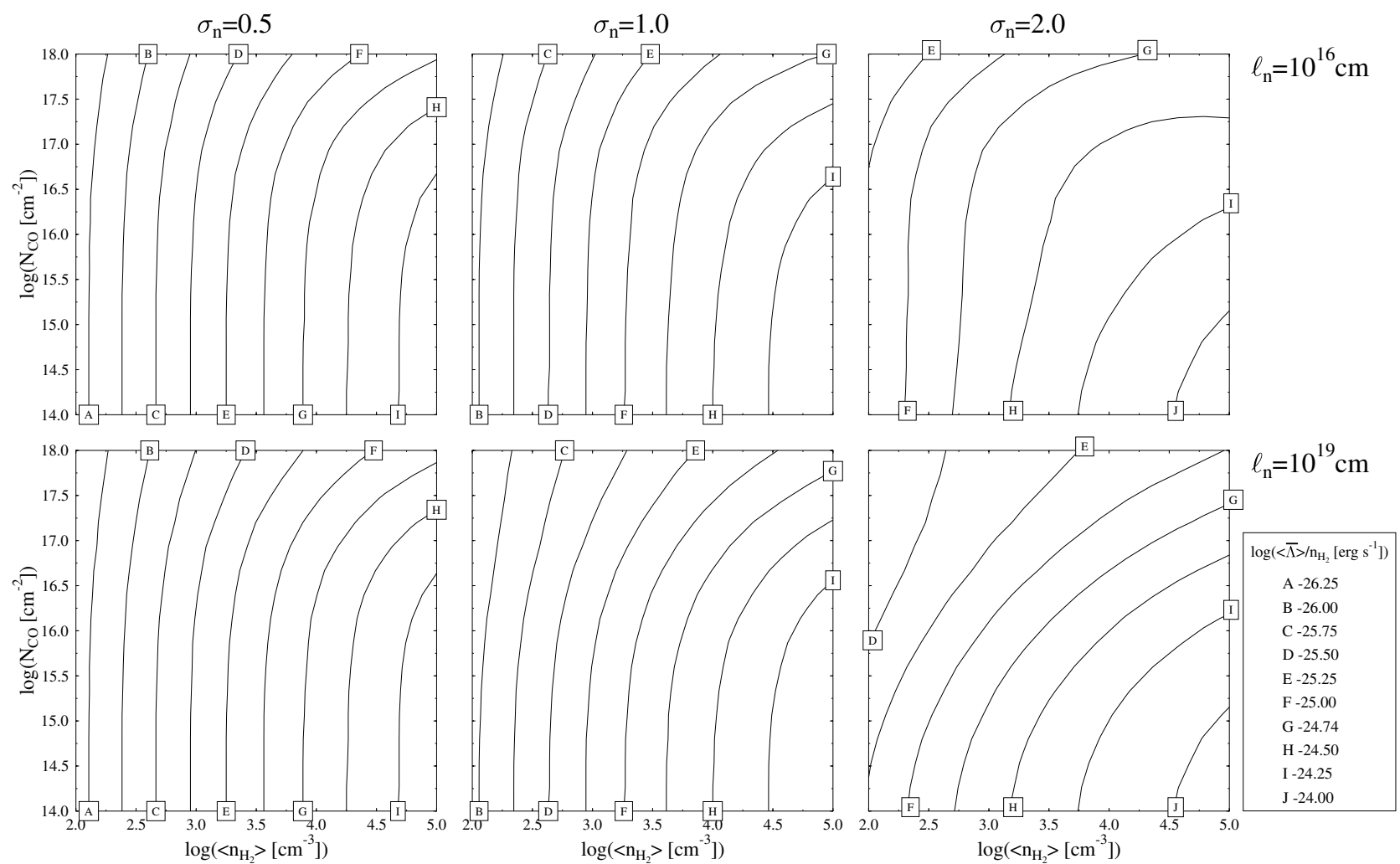

Fig. 9. Volume and density averaged CO cooling rates for a large set of clouds (model B) with different masses and densities. The cooling rate is given as a function of the mean cloud's density and the $\mathrm{CO}$ column density along the cetral line of sight. From the left to the right, $\sigma_{\mathrm{n}}$ is increased fro 0.5 to 2.0. The turbulent velocity is $\sigma_{\mathrm{v}}=0.5 \mathrm{~km} \mathrm{~s}^{-1}$ and the gas temperature is $T_{\text {gas }}=40 \mathrm{~K}$ in all models. 

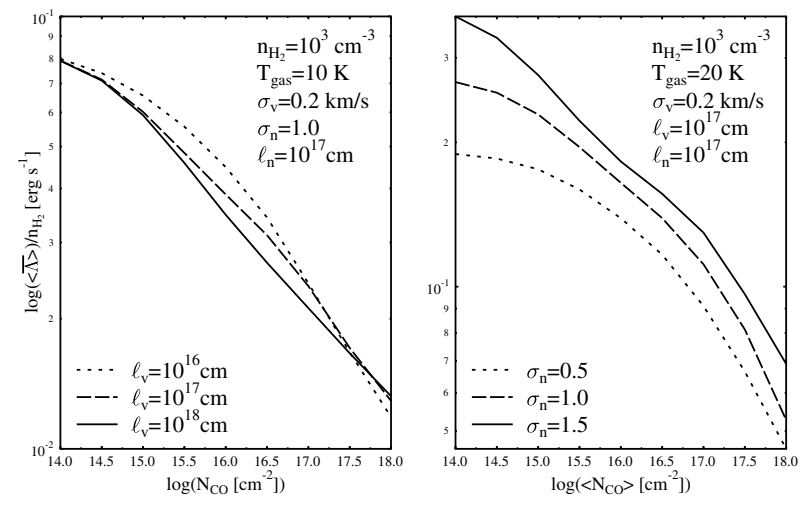

Fig. 10. Volume, density and velocity averaged $\mathrm{CO}$ cooling rates for a series of clouds with different masses accounting for density and velocity fluctuations (model C). Left: $\sigma_{\mathrm{n}}=1.0$ for all graphs while $\ell_{\mathrm{v}}$ is varied. Right: $\ell_{\mathrm{v}}=10 \times 10^{17} \mathrm{~cm}$ for all graphs and $\sigma_{\mathrm{n}}$ is varied.
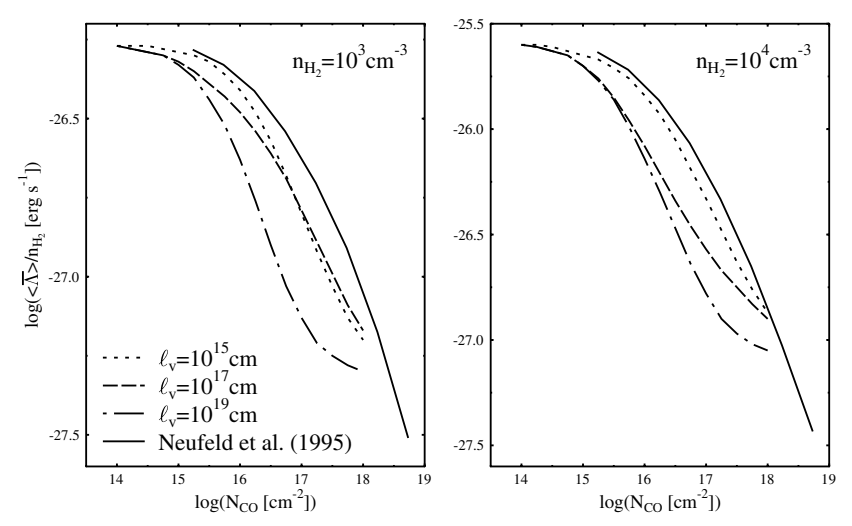

Fig. 11. Comparison of the model A results (homogeneous density distribution) with those of Neufeld et al. (1995). For constant $T_{\text {gas }}=10 \mathrm{~K}$, $\sigma_{\mathrm{v}}=0.2 \mathrm{~km} \mathrm{~s}^{-1}, n_{\mathrm{H}_{2}}=10^{3} \mathrm{~cm}^{-3}$ and $n_{\mathrm{H}_{2}}=10^{4} \mathrm{~cm}^{-3}$, respectively, the volume and velocity averaged cooling rates are given as a function of the CO column density along the central line of sight of each cloud.
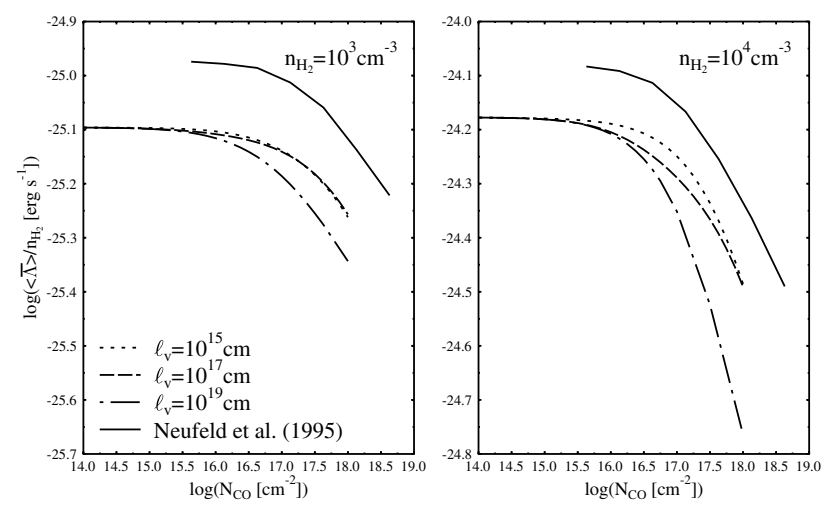

Fig. 12. Comparison of the model A results (homogeneous density distribution) with those of Neufeld et al. (1995). For constant $T_{\text {gas }}=100 \mathrm{~K}$, $\sigma_{\mathrm{v}}=0.5 \mathrm{~km} \mathrm{~s}^{-1}, n_{\mathrm{H}_{2}}=10^{3} \mathrm{~cm}^{-3}$ and $n_{\mathrm{H}_{2}}=10^{4} \mathrm{~cm}^{-3}$, respectively, the volume and velocity averaged cooling rates are given as a function of the CO column density along the central line of sight of each cloud.

following set of model parameters was used: $\left\langle n_{\mathrm{H}_{2}}\right\rangle=320 \mathrm{~cm}^{-3}$, $T_{\text {gas }}=10 \mathrm{~K}, \sigma_{\mathrm{v}}=2.1 \mathrm{~km} \mathrm{~s}^{-1}, R=3.9 \mathrm{pc}, n_{\mathrm{CO}} / n_{\mathrm{H}_{2}}=5 \times 10^{-5}$. The radius $R$ of our model cloud was taken such that its volume equals $L^{3}$. The values $\sigma_{\mathrm{n}}, \ell_{\mathrm{n}}$, and $\ell_{\mathrm{v}}$ characterizing the turbulent velocity and density field were varied until the stochastic

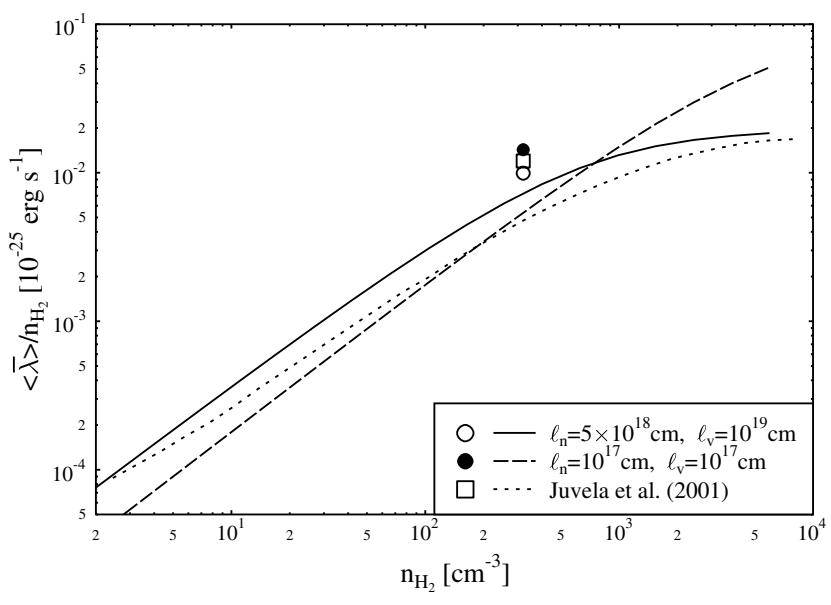

Fig. 13. Comparison of the model $\mathrm{C}$ results (density and velocity fluctuations) with those of Juvela et al. (2001). The lines give the cooling rates as a function of the local density, whereas the square and the circles represent the volume and density averaged cooling rates. The cloud parameters are: $T_{\text {gas }}=10 \mathrm{~K}, \sigma_{\mathrm{v}}=2.1 \mathrm{~km} \mathrm{~s}^{-1},\left\langle n_{\mathrm{H}_{2}}\right\rangle=320 \mathrm{~cm}^{-3}$, and $\sigma_{\mathrm{n}}=1.5$.

radiative transfer results matched the results from Juvela et al. as closely as possible. We found best agreement between the two models for $\sigma_{\mathrm{n}}=1.5, \ell_{\mathrm{n}}=5 \times 10^{18} \mathrm{~cm}$, and $\ell_{\mathrm{v}}=10^{19} \mathrm{~cm}$ (solid line). The conditional expectation value of the cooling rate rises steeply with increasing density but the curve flattens around $n_{\mathrm{H}_{2}} \gtrsim 500 \mathrm{~cm}^{-3}$. For comparison, the conditional expectation value of the cooling rate is also given for a set of smaller correlation lengths in Fig. 13: $\ell_{\mathrm{n}}=10^{17} \mathrm{~cm}$ and $\ell_{\mathrm{v}}=10^{17} \mathrm{~cm}$ (dashed line). For smaller correlation lengths, the discrepancy between the two models increases. Since the mean clump size decreases with decreasing $\ell_{\mathrm{n}}$, much higher densities must be reached, before the high density regions become optically opaque. As a consequence, the flattening of the dashed curve in Fig. 13 sets in at higher densities than for the solid curve. In addition to the local cooling rates, Fig. 13 gives also the overallexpectation value of the cooling rate, i.e. the density, velocity, and volume averaged cooling rate. For $\sigma_{\mathrm{n}}=1.5$ and $\ell_{\mathrm{n}}=10^{19} \mathrm{~cm}$, almost the same average value for the cooling rate is obtained for our stochastic radiative transfer model and the model of Juvela et al. Such large values for $\sigma_{\mathrm{n}}=1.5$ and $\ell_{\mathrm{n}}$ are not unexpected. In the MHD simulations of Padoa \& Nordlund (1999), the medium fragments rapidly into filaments, approximately aligned with the magnetic field, and clumps with very large density contrast. Since these filaments are extremely elongated, large correlation lengths $\ell_{\mathrm{n}}$ and $\ell_{\mathrm{v}}$ in direction of the the magnetic field lines can be expected.

\section{Summary}

Based on a stochastic radiative transfer model (SRTM) which accounts for density and velocity fluctuations with a finite correlation length, we have obtained $\mathrm{CO}$ cooling rates for a molecular gas at temperatures $T_{\text {gas }}=(10-100) \mathrm{K}$ and densities $n_{\mathrm{H}_{2}}=$ $\left(10^{2}-10^{6}\right) \mathrm{cm}^{-3}$. We find the following results:

1. A finite correlation length of the turbulent velocity field tends to decrease the $\mathrm{CO}$ cooling efficiency. Except for a low density medium, $n_{\mathrm{H}_{2}}<10^{3} \mathrm{~cm}^{-3}$, and high column densities, the mean $\mathrm{CO}$ cooling rates are largest for the microturbulent case $\ell_{\mathrm{v}} \rightarrow 0$ and decrease with increasing correlation length of the velocity field. 
2. The results of our model A (turbulent velocity field with finite correlation length, homogeneous density distribution) were compared to the results of Neufeld et al. (1995), which have been obtained from an escape probability formalism. Best agreement for the volume averaged cooling rates is achieved for a small correlation length. With increasing correlation length our cooling efficiency starts to drop and the difference between the two models become substantially.

3. Stochastic density fluctuations tend to increase the $\mathrm{CO}$ cooling efficiency noticeably. For an inhomogeneous stochastic density distribution, cooling by the high rotational lines of $\mathrm{CO}$ is substantially enhanced. Most of the radiation is emitted from regions in the cloud being denser than the average. In addition, an inhomogeneous density field reduces photon trapping which leads to a further increase of the cooling rate.

4. The results of our model $\mathrm{C}$ (combined stochastic density and velocity fluctuations) were compared with the results of Juvela et al. (2001), which were also based on an inhomogeneous clumpy cloud model. We found satisfactory agreement between the two models for rather large correlation lengths of the density and velocity field. For a small correlation length of the density field we found $\mathrm{CO}$ cooling rates much larger than predicted by Juvela at al. (2001).

Acknowledgements. We are grateful to the anonymous referee for the useful comments received. This work was supported by the DFG in the frame of the Sonderforschungsbereich 555 (Teilprojekt B8).

\section{References}

Albrecht, M. A., \& Kegel, W. H. 1987, A\&A, 176, 317

Black, J. H., \& Willner, S. P. 1984, ApJ, 279, 673

Blitz, L. 1987, in Physical Processes in Interstellar Clouds, ed. G. M. Morfill, \& M. Scholer (Reidel Verlag)

Flower, D. R. 2001, J. Phys. B, 34, 2731

Flower, D. R., Launay, J. M. 1985, MNRAS, 214, 271

Gail, H.-P., Hundt, E., Kegel, W. H., Schmid-Burgk, J., \& Traving, G. 1974, A\&A, 32, 65

Gail, H.-P., Sedlmayr, E., \& Traving, G. 1975, A\&A, 44, 421

Gail, H.-P., Sedlmayr, E., \& Traving, G. 1980, JQSRT 23, 267

Goldreich, P., \& Kwan, J. 1974, ApJ, 189, 441

Goldsmith, P. F., \& Langer, W. D. 1978, ApJ, 222, 881

Hegmann, M., \& Kegel, W. H. 2000, A\&A, 359, 405

Hegmann, M., \& Kegel, W. H. 2003, MNRAS, 342, 453

Juvela, M., Padoan, P., \& Nordlund, A 2001, ApJ, 563, 853

Kegel, W. H., Piehler, G., \& Albrecht, M. A. 1987, A\&A, 270, 407

Köppen, J., \& Kegel, W. H. 1980, A\&A 42, 59

Millar, T. J., Farquhar, P. R. A., \& Willacy, K. 1997, A\&AS, 121, 139

Neufeld, D. A., \& Kaufman, J. 1993, ApJ, 418, 263

Neufeld, D. A., Lepp, S., \& Melnick, G. J. 1995, ApJS, 100, 132

Ossenkopf, V., Esquivel, A., Lazarian, A., \& Stutzki, J. 2006, A\&A, 452, 223

Padoan, P., \& Nordlund, A 1999, ApJ, 526, 279

Piehler, G., \& Kegel, W. H. 1995, A\&A, 297, 841

Schöier, F. L., van der Tak, F. F. S., van Dishoeck, E. F., \& Black, J. H. 2005, A\&A, 432, 369

Sobolev, V. V. 1960, Moving envelopes of stars (Cambridge: Harvard University Press)

Spitzer, L. 1985, Physical Processes in the Interstellar Medium (New York: Wiley-Interscience)

Sun, K., Kramer, C., Ossenkopf, V., et al. 2006, A\&A, 451, 539

Viscuso, P. J., \& Chernoff, D. F. 1988, ApJ, 327, 364 